\title{
An update on the next-to-leading order Monte Carlo MCFM
}

\author{
R. K. Ellis ${ }^{\mathrm{a}}$ \\ ${ }^{a}$ Fermilab, PO Box 500, Batavia, IL 60510, USA \\ and \\ CERN, 1211 Geneva 23, Switzerland
}

The current status of the parton level, next-to-leading order Monte Carlo program MCFM is described.

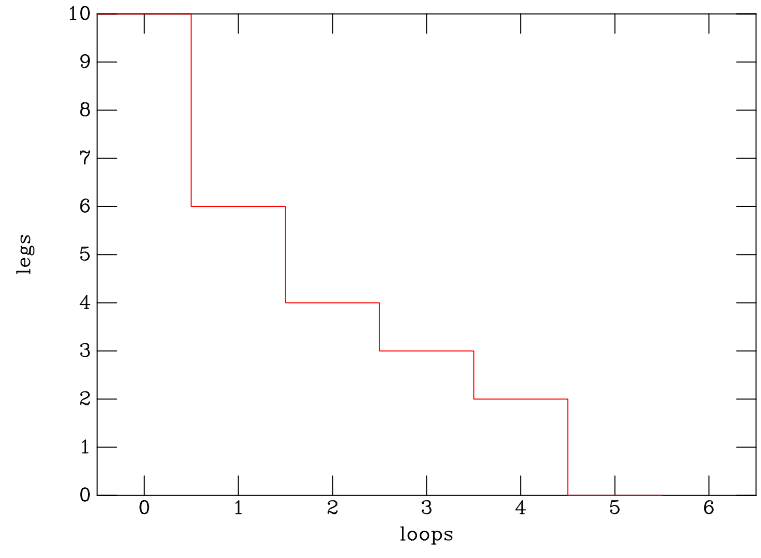

Figure 1. Schematic description of QCD calculations, showing the current status in terms of the number of legs or loops calculated.

\section{Loopverein or Beinverein?}

As illustrated in Fig. 1, the state of the art for radiative corrections to hard processes allows the calculation of graphs with many legs at a low number of loops, or with many loops at a low number of legs, but not both. At LHC, the trend of the phenomenological interest is towards processes with large numbers of legs. It will therefore be important to calculate multi-leg processes at NLO. In fact, the most phenomenologically interesting processes involve vector bosons, leptons, missing energy, or heavy flavours. In addition, many processes can contribute to the same sig- nature, which argues for one (or better, several) unified NLO approaches.

\section{What is MCFM?}

MCFM[1-11] is a NLO parton-level event integrator for many processes. In line with the statement of physics interest given above, it includes processes involving heavy quarks, vector and Higgs bosons, and missing energy. Spin correlations are included in decay of the bosons. Since it is a Monte Carlo program, the distributions of all variables are available. A rough list of the included processes is given in Table 1. A complete list is given in the documentation distributed with the code[1].

Most processes are included at NLO in $\alpha_{S}$, with all the attendant benefits. First, there is a reduced dependence on unphysical scales, leading to a better estimate of rates for physical processes. Second, there is more than one parton in a jet, giving (an albeit primitive) structure to the jet. In addition, for well separated jets, as would occur in the decay of a heavy object, MCFM is expected to give a better estimate than a calculation based on a parton shower. Additionally, many processes are included in a unified framework, allowing easy comparison between processes. The program can be used at the Tevatron, LHC or RHIC by choosing the appropriate energy and parton distribution set.

\subsection{MCFM v. 5.1}

MCFM v.5.1 was released in June 2006 and is available for download[1]. The new NLO pro- 


\begin{tabular}{|ll|}
\hline$p \bar{p} \rightarrow W^{ \pm} / Z$ & $p \bar{p} \rightarrow W^{+}+W^{-}$ \\
$p \bar{p} \rightarrow W^{ \pm}+Z$ & $p \bar{p} \rightarrow Z+Z$ \\
$p \bar{p} \rightarrow W^{ \pm}+\gamma$ & $p \bar{p} \rightarrow W^{ \pm} / Z+H$ \\
$p \bar{p} \rightarrow W^{ \pm}+g^{\star}(\rightarrow b \bar{b})$ & $p \bar{p} \rightarrow Z b \bar{b}$ \\
$p \bar{p} \rightarrow Z b$ & $p \bar{p} \rightarrow W b$ \\
$p \bar{p} \rightarrow Z b j$ & $p \bar{p} \rightarrow W b j$ \\
$p \bar{p} \rightarrow W^{ \pm} / Z+1$ jet & $p \bar{p} \rightarrow W^{ \pm} / Z+2$ jets \\
$p \bar{p}(g g) \rightarrow H$ & $p \bar{p}(g g) \rightarrow H+1$ jet \\
$p \bar{p}(V V) \rightarrow H+2$ jets & $p \bar{p} \rightarrow t \bar{t}$ \\
$p \bar{p} \rightarrow t X(s, t$-channel $)$ & $p \bar{p} \rightarrow t+W$ \\
\hline
\end{tabular}

Table 1

MCFM processes

cesses added in version five are, $(f \equiv q, \bar{q}, g$, generic parton)

$f+f \rightarrow W^{ \pm}+t$

$f+b \rightarrow Z^{0}+b+f$

$f+c \rightarrow Z^{0}+c+f$

The following processes have been added in LO

$f+f \rightarrow Z^{0}\left(\rightarrow e^{-}+e^{+}\right)+c+c$

$f+f \rightarrow t \bar{t}+g$

$f+f \rightarrow H+f+f+f[$ in heavy top limit]

$f+f \rightarrow W^{-}\left(\rightarrow e^{-}+\nu\right)+t+b[$ massive $\mathrm{b}]$

\subsection{Shortcomings of MCFM}

The program MCFM is not a fully-fledged Monte Carlo program, because it lacks a full parton shower and a model for the hadronization of the resultant partons into the observed hadrons. Because it does not produce hadrons it cannot be used as the input to a dectector simulation. Moreover it has both positive and negative weights. MCFM should be rather considered as a parton level integrator.

Remarkable progress has been made in combining NLO calculations with a parton shower (MC@NLO), in such a way that the $O\left(\alpha_{S}\right)$ expansion of the resultant answer agrees with the exact calculation [12]. No attempt has yet been made to extend MCFM in this way. We only note here that an understanding of NLO is a necessary prerequisite for MC@NLO.

In addition there is no inclusion of pure QCD processes, such as $g g \rightarrow g g, g g \rightarrow g g g$ and $g g \rightarrow$ gggg. These processes have been treated by other

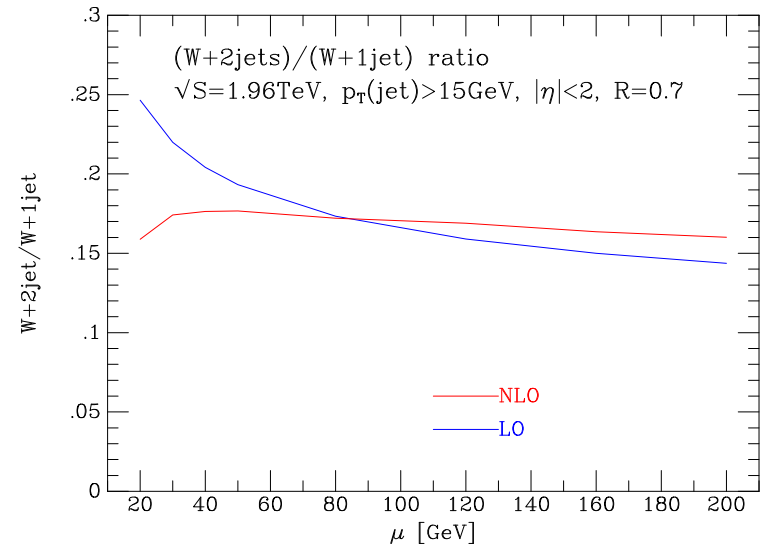

Figure 2. The ratio $(W+2$ jets $) /(W+1$ jet 2$)$ calculated at NLO.

\begin{tabular}{|l|l|l|}
\hline Process & Tevatron[pb], & LHC $[\mathrm{pb}]$ \\
\hline$t \bar{t}$ & 6 & 720 \\
$t \bar{b}$ s-channel & 0.8 & 10 \\
$t \bar{b}$ t-channel & 1.8 & 240 \\
$W t$ & 0.14 & 66 \\
\hline \multicolumn{2}{|l}{ Table 2}
\end{tabular}

Total cross sections for top production

authors $[13,14]$.

Note also that there was an error in previous versions for the cross sections for $W+1$ jet, $Z+1$ jet, leading to $\sim 15 \%$ errors at NLO. This has been corrected in version 5 . An updated figure for the ratio of $W+2$ jets $/ W+1$ jet is given in Fig. 2.

\section{Top production rates}

Table 2 gives the total NLO cross-sections for $t \bar{t}$ production both at the Tevatron and the LHC $[9$, 11]. The total single top cross-section is smaller than the $t \bar{t}$ rate by about a factor of two, at both machines.

\subsection{Backgrounds for single top}

The background processes shown in Fig. 3 are calculated with MCFM at $\sqrt{s}=1.96 \mathrm{TeV}$. The results are given given in Table 3 . With the same 

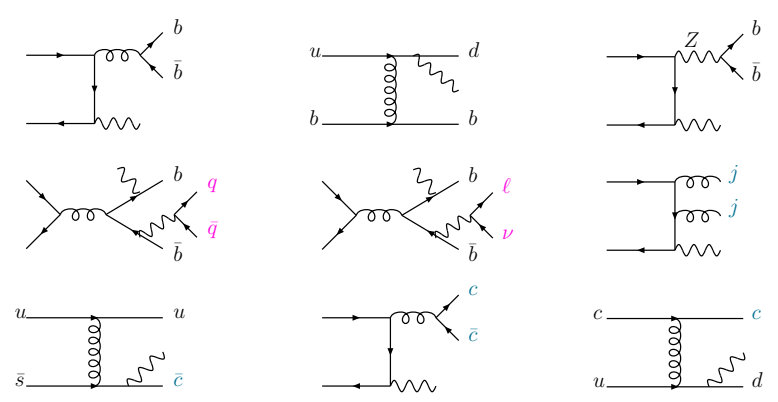

Figure 3. Background processes for single top production

\begin{tabular}{|l|c|}
\hline Process & $\sigma[\mathrm{fb}]$ \\
\hline$q \bar{q} \rightarrow W+b+b$ & 30 \\
$q \bar{q} \rightarrow W+g+g$ & 35 \\
$u s \rightarrow W+u+c$ & 19 \\
$u b \rightarrow W+d+b$ & 11 \\
$q \bar{q} \rightarrow W+c+\bar{c}$ & 6 \\
$u c \rightarrow W+d+c$ & 3 \\
$q \bar{q} \rightarrow W+Z(b \bar{b})$ & 3 \\
$q \bar{q} \rightarrow t \bar{t} \rightarrow W b \bar{b} q \bar{q}$ & 6 \\
$q \bar{q} \rightarrow t \bar{t} \rightarrow W b \bar{b} l \nu$ & 3 \\
\hline
\end{tabular}

Table 3

Cross-sections in $\mathrm{fb}$ include nominal tagging efficiences and mis-tagging/fake rates taken from ref. [11]

set of cuts, the signal rates are $7 \mathrm{fb}$ and $11 \mathrm{fb}$ for $s$ - and $t$-channel respectively. With our nominal efficiencies, the ratio of signal:background is about $1: 6$. For the cuts and efficiencies used we refer the reader to Tables VI,VIII of ref. [11].

Figure 4 shows the $H_{T}$ distribution, both for the signal and the background, where $H_{T}$ is defined as the scalar sum of jet, lepton and missing $E_{T}$. Current estimates are that it will take 1.5 $\mathrm{fb}^{-1}$ to have $3 \sigma$ evidence for single top from a single experiment at the Tevatron[15].

\section{2. $W t$ production}

The last of the single top processes, the $W t$ process which is important at LHC, but negligible at the Tevatron was included in MCFM[11].

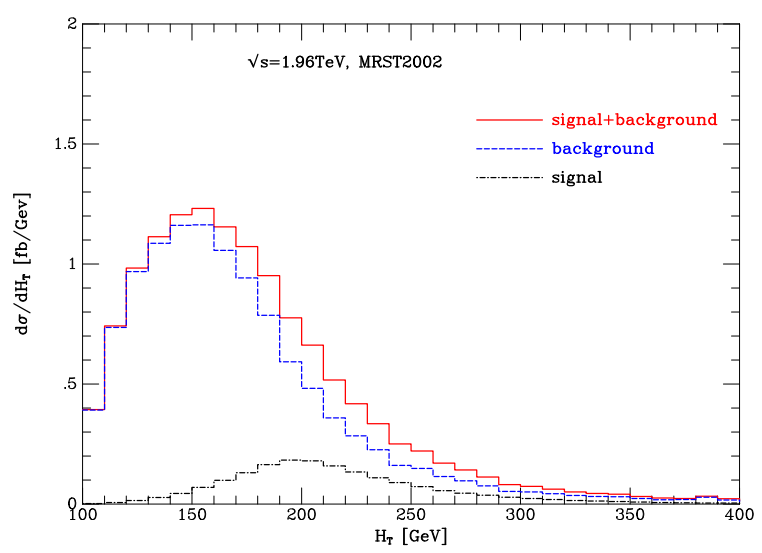

Figure 4. $H_{T}$ for signal and background
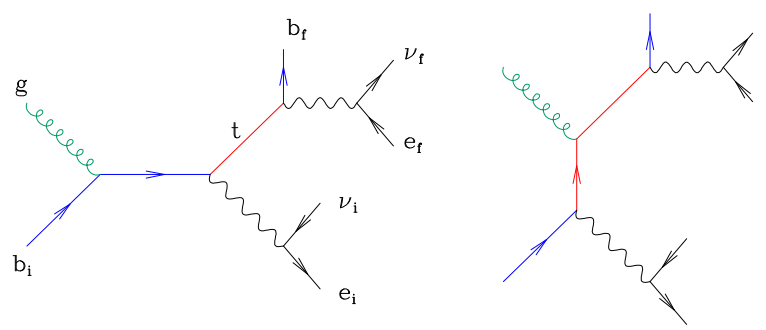

Figure 5. Lowest order diagrams for $W t$ production

The rate depends on $b$-quark distribution in the proton, which is quite poorly known. The top quark, (shown in Fig. 5 in red) is taken onshell, but all spin correlations are retained. Including real radiation we obtain both diagrams with and without a resonant $\bar{t}$ propagator. The former are properly considered as lowest order contributions to $t \bar{t}$ production, whereas the latter are contributions to single top production. We apply a veto on the $p_{T}$ of the additional $\bar{b}$ quark which appears in NLO. If we choose factorization scale $\mu_{F}$ of the same order as the maximum $p_{T}$ which is allowed, we find that when the $p_{T}>\mu_{F}$, the doubly resonant diagrams dominate and a better description is obtained by using the $t \bar{t}$ process. This region 


\begin{tabular}{|l|c|}
\hline Process & $\sigma[\mathrm{fb}]$ \\
\hline$H \rightarrow W W\left(m_{H}=155 \mathrm{GeV}\right)$ & 58.1 \\
continuum WW & 270.5 \\
$t \bar{t}$ & 43.9 \\
$W t$ & 40.1 \\
\hline Table 4
\end{tabular}

Cross sections for $W W$ signal and backgrounds

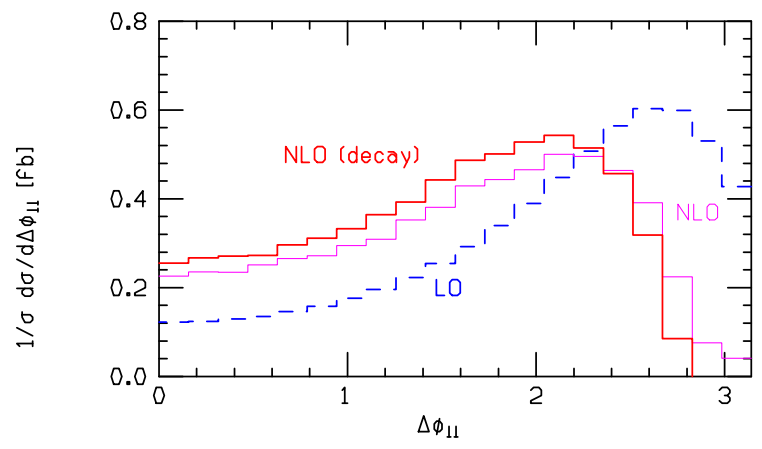

Figure 6. The modification of the DittmarDreiner angle by the inclusion of NLO corrections.

is hence excluded from the single top sample.

The $W t$ background to $H \rightarrow W W^{\star}$ can be estimated using this calculation. The results $[11,23]$ are given in Table 4 . We note that, with the cuts used, the $t \bar{t}$ and $W t$ backgrounds are of similar size.

From Fig. ?? we see that shape of contribution of $W t$ process to Dittmar-Dreiner angle[22] modified at next-to-leading order.

\section{Higgs boson +2 jets}

Although not yet included in MCFM, the calculation of the Higgs +2 -jet rate performed using the effective coupling to gluons is a possible line of development for MCFM. The effective coupling, which is valid in the $m_{t} \rightarrow \infty$ limit, includes a finite radiative correction[20,21].

$\mathcal{L}_{\mathrm{eff}}=\frac{1}{4} A(1+\Delta) H G_{\mu \nu}^{a} G^{a \mu \nu}, \quad \Delta=\frac{11 g^{2}}{16 \pi^{2}}$.
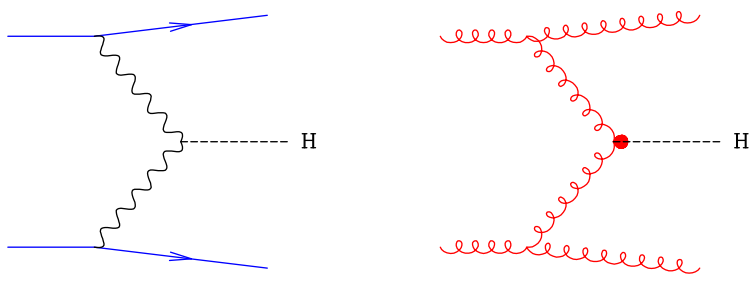

Figure 7. Diagrams for Higgs boson production

$G_{\mu \nu}^{a}$ is the gluon field strength and $H$ is the Higgs-boson field. The effective coupling $A$ is given by

$A=\frac{g^{2}}{12 \pi^{2} v}$,

where $g$ is the strong coupling and $v$ is the vacuum expectation value parameter, $v^{2}=\left(G_{F} \sqrt{2}\right)^{-1}=$ $(246 \mathrm{GeV})^{2}$.

\section{1. $H+2$ jet calculation}

NLO corrections to $W$-fusion mechanism have already been calculated by many authors[16]. All the elements are in place for a full NLO Higgs +2 jets calculation via gluon fusion mechanism, namely the Born level calculation Higgs +4 partons, the real calculation Higgs +5 partons[17], the irtual calculation[19], and the subtraction terms.

\section{2. $H+2$ jet results}

The virtual corrections are included in our code using a seminumerical procedure. We define jets with $p_{t}(j e t)>20 \mathrm{GeV},\left|y_{j}\right|<5, R_{j j}>0.6$ and we vary renormalization and factorization scale together. Our preliminary results indicate the Higgs +2 jet inclusive rate is the better behaved at NLO than the rate for Higgs $+\mathrm{X}$ rate, or Higgs +1 jet+X. Fig. 8 also suggests that a relatively high scale $\mu \sim m_{H}$ is appropriate for the Higgs. When we impose cuts to enhance vector boson fusion, (without central jet veto) we obtain a similar pattern for the $\mu$ dependence as shown in Fig.8. 


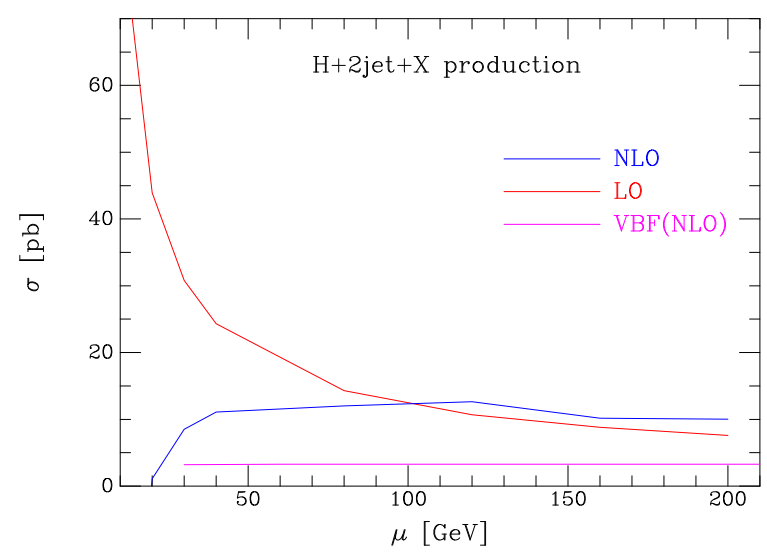

Figure 8. Factorization and renormalization scale dependence of the Higgs +2 jet cross section. Also shown is the result from the VBF process.

\section{Conclusions}

The current release of MCFM (version 5.1, June, 2006) includes the new processes, $p p \rightarrow W t$ and $p p \rightarrow Z b j$ and is available for download[1]. The benefits of a unified approach are beginning to be observed. For example, MCFM allows the calculation of both signal and background for single top production, predominantly at NLO.

I have also presented preliminary results for Higgs +2 jets, calculated at NLO using an effective Lagrangian. The results indicate that a stable result for Higgs +2 jets can be obtained. This is important for estimating the 'background' to the vector boson fusion Higgs production mode. More importantly, it establishes the viability of semi-numerical method for calculating virtual corrections for a real physical process.

\section{REFERENCES}

1. http://mcfm.fnal.gov

2. R. K. Ellis and S. Veseli, Phys. Rev. D 60, 011501 (1999) [arXiv:hep-ph/9810489].

3. J. M. Campbell and R. K. Ellis, Phys. Rev. D 60 (1999) 113006 [arXiv:hep-ph/9905386].

4. J. M. Campbell and R. K. Ellis, Phys. Rev.
D 62 (2000) 114012 [arXiv:hep-ph/0006304].

5. J. Campbell, R. K. Ellis, F. Maltoni and S. Willenbrock, Phys. Rev. D 67 (2003) 095002 [arXiv:hep-ph/0204093].

6. J. Campbell and R. K. Ellis, Phys. Rev. D 65 (2002) 113007 [arXiv:hep-ph/0202176].

7. J. Campbell, R. K. Ellis, F. Maltoni and S. Willenbrock, Phys. Rev. D 69 (2004) 074021 [arXiv:hep-ph/0312024].

8. J. Campbell, R. K. Ellis and D. L. Rainwater, Phys. Rev. D 68 (2003) 094021 [arXiv:hep$\mathrm{ph} / 0308195]$.

9. J. Campbell, R. K. Ellis and F. Tramontano, Phys. Rev. D 70 (2004) 094012 [arXiv:hep$\mathrm{ph} / 0408158]$.

10. J. Campbell, R. K. Ellis, F. Maltoni and S. Willenbrock, Phys. Rev. D 73 (2006) 054007 [arXiv:hep-ph/0510362].

11. J. Campbell and F. Tramontano, Nucl. Phys. B 726 (2005) 109 [arXiv:hep-ph/0506289].

12. S. Frixione and B. R. Webber, arXiv:hepph/0601192.

13. Z. Nagy, Phys. Rev. D 68 (2003) 094002 [arXiv:hep-ph/0307268].

14. W. B. Kilgore and W. T. Giele, Phys. Rev. D 55 (1997) 7183 [arXiv:hep-ph/9610433].

15. A. Gresele, arXiv:hep-ex/0605041.

16. T. Han, G. Valencia and S. Willenbrock, Phys. Rev. Lett. 69, 3274 (1992) [arXiv:hepph/9206246].

17. V. Del Duca, A. Frizzo and F. Maltoni, JHEP 0405 (2004) 064 [arXiv:hep-ph/0404013].

18. R. K. Ellis, W. T. Giele and G. Zanderighi, Phys. Rev. D 73 (2006) 014027 [arXiv:hep$\mathrm{ph} / 0508308]$.

19. R. K. Ellis, W. T. Giele and G. Zanderighi, Phys. Rev. D 72 (2005) 054018 [arXiv:hep$\mathrm{ph} / 0506196]$,

G. Zanderighi, these proceedings.

20. A. Djouadi, M. Spira and P. M. Zerwas, Phys. Lett. B 264 (1991) 440.

21. S. Dawson, Nucl. Phys. B 359, 283 (1991).

22. M. Dittmar and H. K. Dreiner, Phys. Rev. D 55 (1997) 167 [arXiv:hep-ph/9608317].

23. T. Binoth, M. Ciccolini, N. Kauer and M. Kramer, JHEP 0503, 065 (2005) [arXiv:hep-ph/0503094]. 